九州大学学術情報リポジトリ

Kyushu University Institutional Repository

\title{
BEST UNBIASED IN RANDOM EFFECT MODELS
}

Furukawa, Nagata

Kumamoto University | Kyushu University

https://doi.org/10.5109/13013

出版情報：統計数理研究. 11 (1/2)，pp.43-61，1964-03. Research Association of Statistical Sciences バージョン：

権利関係 : 


\title{
BEST UNBIASED ESTIMATES IN RANDOM EFFECT MODELS
}

\author{
By \\ Nagata FURUKAWA
}

(Received December 20, 1963)

\section{§. Introduction.}

In this paper we shall be concerned with the point estimation of the parameters in the random effect models for the complete multi-way layouts and the nested designs. This paper gives the exact formulae of sufficient statistics and those of the best unbiased estimates of parameters. "The best unbiased estimate", here, implies the one having the minimum variance of all unbiased estimates.

F. A. Graybill and A. W. Wortham [4] pointed out that in random effect model for the multi-way layouts the usual estimates for variance components were the best unbiased estimates, but it contained an error in enumerating the sufficient statistics. S. N. Roy and R. Gnanadesikan [10] showed a similar result supporting the above result, but the dimensionality of the parameter space was ignored and some additional conditions concerning the functional relationship among the parameters seem to the author to be need for their result to hold valid. In this sense we have had to start anew independently from these authors in order to establish the results in this paper.

F. A. Graybill and R. A. Hultquist [3] obtained a sufficient condition for the existence of a complete set of sufficient statistics in random effect model. Our emphasis in this paper is however placed not only on the existence of a complete set of sufficient statistics but also on the exact formulae of the sufficient statistics and those of the best unbiased estimates.

The recent results of D. L. Weeks and F. A. Graybill [11] are concerned with three factor effect. Neither their results nor ours do contain the others and it is noted that they are exclusively concerned with minimal sufficient statistics without any regard to a complete set of sufficient statistics.

\section{§ 2. Preliminaries.}

Let $\boldsymbol{A}=\left(a_{i j}\right), \boldsymbol{B}=\left(b_{i j}\right)$, then the Kronecker product denoted by $\boldsymbol{A} \otimes \boldsymbol{B}$ is defined as the matrix $\left(a_{i j} B\right)$ in the usual way. The Kronecker product of any number of matrices is defined as the natural generalization of two 
matrices, and we shall write the Kronecker product of $n$ matrices $\boldsymbol{A}_{1}, \boldsymbol{A}_{2}$, $\ldots \boldsymbol{A}_{n}$, as $\prod_{i=1}^{n} \otimes \boldsymbol{A}_{i}$.

In this paper, we shall make use of the well-known relations concerning the Kronecker products of two matrices such as $(\boldsymbol{A} \otimes \boldsymbol{B})(\boldsymbol{C} \otimes \boldsymbol{D})=$ $\boldsymbol{A} \boldsymbol{C} \otimes \boldsymbol{B D},(\boldsymbol{A} \otimes \boldsymbol{B})^{-1}=\boldsymbol{A}^{-1} \otimes \boldsymbol{B}^{-1},(\boldsymbol{A} \otimes \boldsymbol{B})^{\prime}=\boldsymbol{A}^{\prime} \otimes \boldsymbol{B}^{\prime}$, and their generalizations to the products of any number of matrices without mentioning explicitly.

Throughout this paper we shall write the $n \times n$ unit matrix as $\boldsymbol{I}_{n}, \boldsymbol{E}_{n}$ denotes the $n \times n$ matrix with the elements all equal to 1 . Let $\boldsymbol{H}_{n}$ be the $n \times n$ matrix with the elements all equal to zero except for the element of the first row in the first column equal to 1 , and let $\boldsymbol{K}_{n}=\boldsymbol{I}_{n}-\boldsymbol{H}_{n}$, namely,

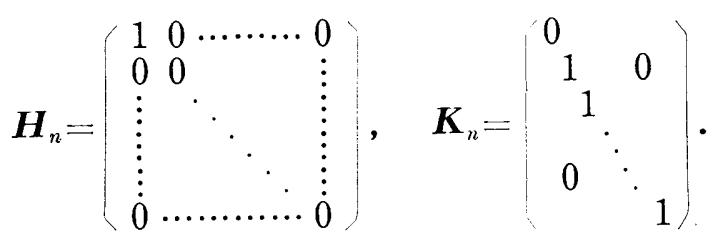

Further let $\boldsymbol{T}_{n}$ be defined as the orthogonal matrix with the elements of the first row all equal to $\frac{1}{\sqrt{ } n}$, namely,

$$
\boldsymbol{T}_{n}=\left(\begin{array}{cccc}
\frac{1}{V \bar{n}} & \frac{1}{V / n} & \cdots \cdots & \frac{1}{\sqrt{n}} \\
\times & \times & \cdots \cdots & \times \\
\vdots & \vdots & & \vdots \\
\vdots & \vdots & & \vdots \\
\times & \times & \cdots \cdots & \times
\end{array}\right)
$$

Then we have easily

$$
\boldsymbol{T}_{n} \boldsymbol{E}_{n} \boldsymbol{T}_{n}^{\prime}=n \boldsymbol{H}_{n} .
$$

\section{$\S 3$. The case of the 2 -way layout.}

This section is devoted to the case of the 2-way layout. Although there is no essential difference between the case of the 2-way layout and the case of the general $r$-way layout, the notation system we need in the latter case is rather cumbersome and complicated that it would be, the author feels, necessary to treat this special case for the preparatory exposition of the basic techniques in the developments of the arguments in the general case.

In this section we shall be concerned with the model equation,

$$
\begin{aligned}
x_{t_{1} t_{2} t_{3}}= & \mu+a\left(1 ; t_{1}\right)+a\left(2 ; t_{2}\right)+a\left(1,2 ; t_{1}, t_{2}\right)+e_{t_{1} t_{2} t_{3}}, \\
& \left(t_{j}=1,2, \cdots, n_{j}, j=1,2,3\right),
\end{aligned}
$$


where $\mu$ is a constant denoting the general mean, and $a\left(1 ; t_{1}\right), a\left(2 ; t_{2}\right)$, $a\left(1,2, ; t_{1}, t_{2}\right)$ and $e_{t_{1} t_{2} t_{3}}$ are distributed normally with mean zero and the variance $\sigma_{1}^{2}, \sigma_{2}^{2}, \sigma_{12}^{2}$ and $\sigma_{\epsilon}^{2}$ respectively, and further they are all independent to each other. The variance-covariance matrix of these $n_{1} n_{2} n_{3}$ variables $x_{t_{1} t_{2} t_{3}}$ are given by

$$
\text { (3.2) } \begin{aligned}
\boldsymbol{V}= & \sigma_{1}^{2} \boldsymbol{I}_{n_{1}} \otimes \boldsymbol{E}_{n_{2}} \otimes \boldsymbol{E}_{n_{3}}+\sigma_{2}^{2} \boldsymbol{E}_{n_{1}} \otimes \boldsymbol{I}_{n_{2}} \otimes \boldsymbol{E}_{n_{3}}+\sigma_{12}^{2} \boldsymbol{I}_{n_{1}} \otimes \boldsymbol{I}_{n_{2}} \otimes \boldsymbol{E}_{n_{3}} \\
& +\sigma_{e}^{2} \boldsymbol{I}_{n_{1}} \otimes \boldsymbol{I}_{n_{2}} \otimes \boldsymbol{I}_{n_{3}} .
\end{aligned}
$$

At first we shall evaluate the determinant of this matrix, which is equal to the determinant of the following matrix,

$$
\left(\boldsymbol{T}_{n_{1}} \otimes \boldsymbol{T}_{n_{2}} \otimes \boldsymbol{T}_{n_{3}}\right) \boldsymbol{V}\left(\boldsymbol{T}_{n_{1}} \otimes \boldsymbol{T}_{n_{2}} \otimes \boldsymbol{T}_{n_{3}}\right)^{\prime} .
$$

In view of $(2.3)$, this is equal to

$$
\begin{aligned}
\boldsymbol{n}_{2} \boldsymbol{n}_{3} \sigma_{1}^{2} & \boldsymbol{I}_{n_{1}} \otimes \boldsymbol{H}_{n_{2}} \otimes \boldsymbol{H}_{n_{3}}+\boldsymbol{n}_{\mathbf{1}} \boldsymbol{n}_{3} \sigma_{2}^{2} \boldsymbol{H}_{n_{1}} \otimes \boldsymbol{I}_{n_{2}} \otimes \boldsymbol{H}_{n_{3}}+\boldsymbol{n}_{3} \sigma_{12}^{2} \boldsymbol{I}_{n_{1}} \otimes \boldsymbol{I}_{n_{2}} \otimes \boldsymbol{H}_{n_{3}} \\
& +\sigma_{e}^{2} \boldsymbol{I}_{n_{1}} \otimes \boldsymbol{I}_{n_{2}} \otimes \boldsymbol{I}_{n_{3}} \\
= & \boldsymbol{n}_{2} \boldsymbol{n}_{3} \sigma_{1}^{2}\left(\boldsymbol{H}_{n_{1}}+\boldsymbol{K}_{n_{1}}\right) \otimes \boldsymbol{H}_{n_{2}} \otimes \boldsymbol{H}_{n_{3}}+\boldsymbol{n}_{1} \boldsymbol{n}_{3} \sigma_{2}^{2} \boldsymbol{H}_{n_{1}} \otimes\left(\boldsymbol{H}_{n_{2}}+\boldsymbol{K}_{n_{2}}\right) \otimes \boldsymbol{H}_{n_{3}} \\
& +\boldsymbol{n}_{3} \sigma_{12}^{2}\left(\boldsymbol{H}_{n_{1}}+\boldsymbol{K}_{n_{1}}\right) \otimes\left(\boldsymbol{H}_{n_{2}}+\boldsymbol{K}_{n_{2}}\right) \otimes \boldsymbol{H}_{n_{3}} \\
& +\sigma_{e}^{2}\left(\boldsymbol{H}_{n_{1}}+\boldsymbol{K}_{n_{1}}\right) \otimes\left(\boldsymbol{H}_{n_{2}}+\boldsymbol{K}_{n_{2}}\right) \otimes\left(\boldsymbol{H}_{n_{3}}+\boldsymbol{K}_{n_{3}}\right) \\
= & \left(\boldsymbol{n}_{2} \boldsymbol{n}_{3} \sigma_{1}^{2}+\boldsymbol{n}_{1} \boldsymbol{n}_{3} \sigma_{2}^{2}+\boldsymbol{n}_{3} \sigma_{12}^{2}+\sigma_{e}^{2}\right) \boldsymbol{H}_{n_{1}} \otimes \boldsymbol{H}_{n_{2}} \otimes \boldsymbol{H}_{n_{3}} \\
& +\left(\boldsymbol{n}_{2} \boldsymbol{n}_{3} \sigma_{1}^{2}+\boldsymbol{n}_{3} \sigma_{12}^{2}+\sigma_{e}^{2}\right) \boldsymbol{K}_{n_{1}} \otimes \boldsymbol{H}_{n_{2}} \otimes \boldsymbol{H}_{n_{3}} \\
& +\left(\boldsymbol{n}_{1} \boldsymbol{n}_{3} \sigma_{2}^{2}+\boldsymbol{n}_{3} \sigma_{12}^{2}+\sigma_{e}^{2}\right) \boldsymbol{H}_{n_{1}} \otimes \boldsymbol{K}_{n_{2}} \otimes \boldsymbol{H}_{n_{3}} \\
& +\left(\boldsymbol{n}_{3} \sigma_{12}^{2}+\sigma_{e}^{2}\right) \boldsymbol{K}_{n_{1}} \otimes \boldsymbol{K}_{n_{2}} \otimes \boldsymbol{H}_{n_{3}}+\sigma_{e}^{2} \boldsymbol{I}_{n_{1}} \otimes \boldsymbol{I}_{n_{2}} \otimes \boldsymbol{K}_{n_{3}} .
\end{aligned}
$$

Thus the matrix (3.3) is expressed as the linear form of five matrices, and as all of them are diagonal, this matrix is also diagonal, and any two matrices have no non-zero element in common. This fact leads us to the evaluation of the determinant as follows,

$$
\begin{aligned}
|\boldsymbol{V}|= & \left(\boldsymbol{n}_{2} \boldsymbol{n}_{3} \sigma_{1}^{2}+\boldsymbol{n}_{1} \boldsymbol{n}_{3} \sigma_{2}^{2}+\boldsymbol{n}_{3} \sigma_{12}^{2}+\sigma_{e}^{2}\right)\left(\boldsymbol{n}_{2} \boldsymbol{n}_{3} \sigma_{1}^{2}+\boldsymbol{n}_{3} \sigma_{12}^{2}+\sigma_{e}^{2}\right)^{\left(n_{1}-1\right)} \\
& \left(\boldsymbol{n}_{1} \boldsymbol{n}_{3} \sigma_{2}^{2}+\boldsymbol{n}_{3} \sigma_{12}^{2}+\sigma_{e}^{2}\right)^{\left(n_{2}-1\right)} \cdot\left(\boldsymbol{n}_{3} \sigma_{12}^{2}+\sigma_{e}^{2}\right)^{\left(n_{1}-1\right)\left(n_{2}-1\right)}\left(\sigma_{e}^{2}\right)^{n_{1} n_{2}\left(n_{3}-1\right)},
\end{aligned}
$$

or by writing

$$
\begin{aligned}
& \theta_{0}=\sigma_{e}^{2}, \\
& \theta_{12}=\boldsymbol{n}_{3} \sigma_{12}^{2}+\sigma_{e}^{2}, \\
& \theta_{1}=\boldsymbol{n}_{2} \boldsymbol{n}_{3} \sigma_{1}^{2}+\boldsymbol{n}_{3} \sigma_{12}^{2}+\sigma_{e}^{2}, \\
& \theta_{2}=\boldsymbol{n}_{1} \boldsymbol{n}_{3} \sigma_{2}^{2}+\boldsymbol{n}_{3} \sigma_{12}^{2}+\sigma_{e}^{2}, \\
& \theta_{E}=\boldsymbol{n}_{2} \boldsymbol{n}_{3} \sigma_{1}^{2}+\boldsymbol{n}_{1} \boldsymbol{n}_{3} \sigma_{2}^{2}+\boldsymbol{n}_{3} \sigma_{12}^{2}+\sigma_{e}^{2},
\end{aligned}
$$


we have finally

$$
\boldsymbol{V} \mid=\theta_{E} \theta_{1}^{\left({ }_{1}-1\right)} \theta_{2}{ }^{\left(n_{2}-1\right)} \theta_{12}{ }^{\left(n_{1}-1\right)\left(n_{2}-1\right)} \theta_{0}{ }^{{ }_{11} n_{2}\left(n_{3}-1\right)} .
$$

Now let us find out the inverse matrix of (3.2). Five matrices in (3.3) are all diagonal matrices with the diagonal elements equal to zero or one, and these have no non-zero element in common. Thus we have

$$
\text { (3.7) } \begin{aligned}
& {\left[\left(\boldsymbol{T}_{n_{1}} \otimes \boldsymbol{T}_{n_{2}} \otimes \boldsymbol{T}_{n_{3}}\right) \boldsymbol{V}\left(\boldsymbol{T}_{n_{1}} \otimes \boldsymbol{T}_{n_{2}} \otimes \boldsymbol{T}_{n_{3}}\right)^{\prime}\right]^{-1} } \\
&=\frac{1}{\theta_{E}} \boldsymbol{H}_{n_{1}} \otimes \boldsymbol{H}_{n_{2}} \otimes \boldsymbol{H}_{n_{3}}+\frac{1}{\theta_{1}} \boldsymbol{K}_{n_{1}} \otimes \boldsymbol{H}_{n_{2}} \otimes \boldsymbol{H}_{n_{3}}+\frac{1}{\theta_{2}^{-}} \boldsymbol{H}_{n_{1}} \otimes \boldsymbol{K}_{n_{2}} \otimes \boldsymbol{H}_{n_{3}} \\
&+\frac{1}{\theta_{12}} \boldsymbol{K}_{n_{1}} \otimes \boldsymbol{K}_{n_{2}} \otimes \boldsymbol{H}_{n_{3}}+\frac{1}{\theta_{0}} \boldsymbol{I}_{n_{1}} \otimes \boldsymbol{I}_{n_{2}} \otimes \boldsymbol{K}_{n_{3}} \\
&= \frac{1}{\theta_{E}} \boldsymbol{H}_{n_{1}} \otimes \boldsymbol{H}_{n_{2}} \otimes \boldsymbol{H}_{n_{3}}+\frac{1}{\theta_{1}}\left(\boldsymbol{I}_{n_{1}}-\boldsymbol{H}_{n_{1}}\right) \otimes \boldsymbol{H}_{n_{2}} \otimes \boldsymbol{H}_{n_{3}} \\
&+\frac{1}{\theta_{2}} \boldsymbol{H}_{n_{1}} \otimes\left(\boldsymbol{I}_{n_{2}}-\boldsymbol{H}_{n_{2}}\right) \otimes \boldsymbol{H}_{n_{3}}+\frac{1}{\theta_{12}}\left(\boldsymbol{I}_{n_{1}}-\boldsymbol{H}_{n_{1}}\right) \otimes\left(\boldsymbol{I}_{n_{2}}-\boldsymbol{H}_{n_{2}}\right) \otimes \boldsymbol{H}_{n_{3}} \\
&+\frac{1}{\theta_{0}} \boldsymbol{I}_{n_{1}} \otimes \boldsymbol{I}_{n_{2}} \otimes\left(\boldsymbol{I}_{n_{3}}-\boldsymbol{H}_{n_{3}}\right) .
\end{aligned}
$$

In view of (2.3), we have

$$
\begin{aligned}
\boldsymbol{V}^{-1} & =\frac{1}{\boldsymbol{n}_{1} \boldsymbol{n}_{2} \boldsymbol{n}_{3} \theta_{E}} \boldsymbol{E}_{n_{1}} \otimes \boldsymbol{E}_{n_{2}} \otimes \boldsymbol{E}_{n_{3}}+\frac{1}{\boldsymbol{n}_{2} \boldsymbol{n}_{3} \theta_{1}}\left(\boldsymbol{I}_{n_{1}}-\frac{1}{\boldsymbol{n}_{1}} \boldsymbol{E}_{n_{1}}\right) \otimes \boldsymbol{E}_{n_{2}} \otimes \boldsymbol{E}_{n_{3}} \\
& +\frac{1}{\boldsymbol{n}_{\mathbf{1}} \boldsymbol{n}_{3} \theta_{2}} \boldsymbol{E}_{n_{1}} \otimes\left(\boldsymbol{I}_{n_{2}}-\frac{1}{\boldsymbol{n}_{2}} \boldsymbol{E}_{n_{2}}\right) \otimes \boldsymbol{E}_{n_{3}} \\
& +\frac{1}{\boldsymbol{n}_{3} \theta_{12}}\left(\boldsymbol{I}_{n_{1}}-\frac{1}{\boldsymbol{n}_{1}} \boldsymbol{E}_{n_{1}}\right) \otimes\left(\boldsymbol{I}_{n_{2}}-\frac{1}{\boldsymbol{n}_{2}} \boldsymbol{E}_{n_{2}}\right) \otimes \boldsymbol{E}_{n_{3}} \\
& +\frac{1}{\theta_{0}} \boldsymbol{I}_{n_{1}} \otimes \boldsymbol{I}_{n_{2}} \otimes\left(\boldsymbol{I}_{n_{3}}-\frac{1}{\boldsymbol{n}_{3}} \boldsymbol{E}_{n_{3}}\right) .
\end{aligned}
$$

Finally, by noting the relations

$$
\boldsymbol{Y}_{n}^{\prime} \boldsymbol{E}_{n} \boldsymbol{Y}_{n}=\left(\sum_{i=1}^{n} \boldsymbol{y}_{i}\right)^{2} \text { and } \boldsymbol{Y}_{n}^{\prime} \boldsymbol{I}_{n} \boldsymbol{Y}_{n}=\sum_{i=1}^{n} \boldsymbol{y}_{i}^{2},
$$

where $\boldsymbol{Y}_{n}^{\prime}$ is any $n$-dimensional vector $\boldsymbol{Y}_{n}^{\prime}=\left(\boldsymbol{y}_{1}, \boldsymbol{y}_{2}, \cdots, \boldsymbol{y}_{n}\right)$, our joint density function is given by

$$
\begin{aligned}
f(\boldsymbol{X}) & =\left(\frac{1}{\sqrt{2 \pi}}\right)^{n_{1} n_{2} n_{3}} \boldsymbol{V}^{-1 / 2} \exp \left[-\frac{1}{2}(\boldsymbol{X}-\boldsymbol{\mu})^{\prime} \boldsymbol{V}^{-1}(\boldsymbol{X}-\boldsymbol{\mu})\right] \\
& =\left(\frac{1}{\sqrt{2 \pi}}\right)^{n_{1} n_{2} n_{3}} \theta_{E}^{-1 / 2} \theta_{1}^{-\left(n_{1}-1\right) / 2} \theta_{2}^{-\left(n_{2}-1\right) / 2} \theta_{12}^{-\left(n_{1}-1\right)\left(n_{1}-1\right) / 2} \theta_{0}^{-n_{1} n_{2}\left(n_{3}-1\right) / 2}
\end{aligned}
$$




$$
\begin{aligned}
\cdot \exp & {\left[-\frac{1}{2}\left\{\frac{n_{1} n_{2} n_{3}}{\theta_{E}}\left(\bar{x}_{\ldots} \ldots-\mu\right)^{2}+{ }_{\theta_{1}} \sum_{t_{1}}\left(\bar{x}_{t_{1} \bullet}-\bar{x}_{\ldots} \ldots\right)^{2}\right.\right.} \\
& +\frac{n_{1} n_{3}}{\theta_{2}} \sum_{t_{2}}\left(\bar{x}_{\bullet t_{2} \bullet}-\bar{x}_{\ldots}\right)^{2}+\frac{n_{3}}{\theta_{12}} \sum_{t_{1}} \sum_{t_{2}}\left(\bar{x}_{t_{1} t_{2} \bullet}-\bar{x}_{t_{1} \bullet}-\bar{x}_{\iota_{2} \bullet}+\bar{x}_{\bullet . .}\right)^{2} \\
& \left.\left.+\frac{1}{\theta_{0}} \sum_{t_{1}} \sum_{t_{2}} \sum_{t_{3}}\left(x_{t_{1} t_{2} t_{3}}-\bar{x}_{t_{1 t_{2}} \bullet}\right)^{2}\right\}\right] .
\end{aligned}
$$

We have the family of distributions whose parameter space is written explicitly as

$$
\Omega=\left(\begin{array}{l}
0 \leqq \theta_{0}<\infty, \quad \theta_{E}=\theta_{1}+\theta_{2}-\theta_{12}, \\
\theta_{0} \leqq \theta_{12}<\infty, \\
\theta_{12} \leqq \theta_{1}<\infty, \quad-\infty<\mu<\infty \\
\theta_{12} \leqq \theta_{2}<\infty,
\end{array}\right),
$$

and whose minimal sufficient statistics are given by the following five statistics

$$
\begin{aligned}
& S_{0}=\sum_{t_{1}} \sum_{t_{2}} \sum_{t_{3}}\left(x_{t_{1} t_{2} t_{3}}-\bar{x}_{t_{1} t_{2}}\right)^{2}, \\
& S_{1}=n_{2} n_{3} \sum_{t_{1}}\left(\bar{x}_{t_{1} \bullet \bullet}-\bar{x}_{\ldots .}\right)^{2}, \\
& S_{2}=n_{1} n_{3} \sum_{t_{2}}\left(\bar{x}_{t_{2} \bullet}-\bar{x}_{\ldots .}\right)^{2}, \\
& S_{12}=n_{3} \sum_{t_{1}} \sum_{t_{2}}\left(\bar{x}_{t_{1} t_{2} \bullet}-\bar{x}_{t_{1} . .}-\bar{x}_{\bullet t_{2} \bullet}+\bar{x}_{\ldots . .}\right)^{2},
\end{aligned}
$$

and

$$
\bar{x} \ldots
$$

Since the family disrtibution of these sufficient statistics is strongly complete in virtue of a lemma of Gautschi [5], the theory of estimation tells us that the usual estimates are the unique minimum variance unbiased estimates of the variance components $\sigma_{e}^{2}, \sigma_{1}^{2}, \sigma_{2}^{2}, \sigma_{12}^{2}$ and the general mean $\mu$.

\section{$\S 4$. The case of the $r$-way layout.}

In this section we shall give the results and the proofs in the case of the $r$-way layout with the model given by

$$
\begin{array}{r}
x_{t_{1} t_{2} \cdots t_{r} t_{r+1}}=\mu+\sum_{k=1}^{r} \sum_{t_{k} \subset R} a\left(i_{1} \cdots i_{k} ; t_{i_{1}}, \cdots, t_{i_{k}}\right)+e_{t_{1} \cdots t_{r} t_{r+1}}, \\
\left(t_{j}=1,2, \cdots, n_{j} ; j=1,2, \cdots, r+1\right),
\end{array}
$$

where $\mu$ is a constant denoting the general mean, $a\left(i_{1}, \cdots, i_{k} ; t_{i_{1}}, \cdots, t_{i_{k}}\right)$ denotes the main effect of the $i_{1}$-th factor if $k=1$, the interaction between $i_{1}$-th, $i_{2}$-th, $\cdots, i_{k}$-th factors with the level $t_{i_{1}}, t_{i_{2}}, \cdots, t_{i_{k}}$ if $k \neq 1$, and $e_{t_{1} \cdots t_{r} t_{r+1}}$ 
denotes the error term. Further, the following distributional assumptions will be made;

(1) $\left\{a\left(i_{1}, \cdots, i_{k} ; t_{i_{1}}, \cdots, t_{i_{k}}\right)\right\}$ is distributed as the multivariate normal, mean vector 0 , covariance matrix $\sigma_{I_{k}}^{2} \boldsymbol{I}_{n_{i_{1}} \cdots n_{i_{k}}}$ for $k=1,2, \cdots, r$;

(2) $\left\{e_{t_{1} \ldots t_{r} t_{r+1}}\right\}$ is distributed as the multivariate normal, mean vector 0 , covariance matrix $\sigma_{e}^{2} I_{n_{1} \cdots n_{r+1}}$;

(3) $\left\{a\left(i_{1}, \cdots, i_{k} ; t_{i_{1}}, \cdots, t_{i_{k}}\right)\right\}$ and $\left\{a\left(j_{1}, \cdots, j_{h} ; t_{j_{1}}, \cdots, t_{j_{h}}\right)\right\}$ are mutually independent for $\left(i_{1}, \cdots, i_{k}\right) \rightleftharpoons\left(j_{1}, \cdots, j_{h}\right)$;

(4) $\left\{a\left(i_{1}, \cdots, i_{k} ; t_{i_{1}}, \cdots, t_{i_{k}}\right)\right\}$ and $\left\{e_{t_{1} \cdots t_{r} t_{r+1}}\right\}$ are mutually independent for $k=1,2, \cdots, r$.

In the above equation (4.1), $R$ denotes the set of integers $(1,2, \cdots, r)$ and $I_{k}$ denotes the subsets $\left(i_{1}, i_{2}, \cdots, i_{k}\right)$ of $R=(1,2, \cdots, r)$ with the relations $i_{1}<i_{2}<\cdots<i_{k} \cdot \sum_{I_{k} \subset R}$ denotes the summation for all subsets $I_{k}$ of size $k$ in $R$.

Throughout this paper the notations such as $I_{k}, J_{h}, L_{p}$ etc. mean sets of integers $\left(i_{1}, i_{2}, \cdots, i_{k}\right),\left(j_{1}, j_{2}, \cdots, j_{h}\right),\left(l_{1}, l_{2}, \cdots, l_{p}\right)$ etc. respectively, and

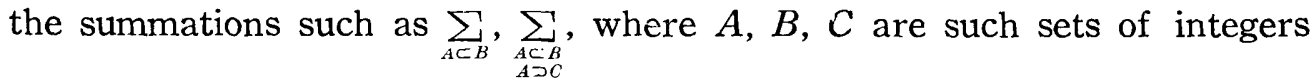
as stated above, mean the sum of all numbers $a_{A}$ 's having $A$ as the suffixes which are included in $B$, or included in $B$ and including $C$, respectively.

Corresponding to (3.2), we have the expression of the variance-covariance matrix in terms of the Kronecker products as follows

$$
\boldsymbol{V}=\sum_{k=1}^{r} \sum_{I_{k} \subset R} \sigma_{I_{k}}^{2}\left[\prod_{j=1}^{r} \otimes\left(\boldsymbol{E}_{n_{j}}^{1-\delta_{I_{k}}^{j}} \boldsymbol{I}_{n_{j}}^{\hat{\boldsymbol{o}}_{I}^{j}}\right)\right] \otimes \boldsymbol{E}_{n_{r+1}}+\sigma_{e}^{2} \boldsymbol{I}_{n_{1}} \otimes \cdots \otimes \boldsymbol{I}_{n_{r+1}},
$$

where $\delta_{I k}^{j}$ is a sort of generalization of the Kronecker's delta which is

$$
\delta_{I_{k}}^{j}= \begin{cases}1 & \text { if } j \text { is equal to either member of } I_{k}, \\ 0 & \text { otherwise, }\end{cases}
$$

and $\boldsymbol{E}^{\circ}$ is defined to be a unit matrix $I$.

For the developments of the arguments in this section we have to prepare with a number of notations and lemmas as follows.

Definition 4. 1.

$$
\begin{aligned}
& A_{\left(I_{k}\right)}=\sum_{p=k}^{r} \sum_{\substack{L_{p} \supset I_{k} \\
L_{p} \subset R}} \sigma_{L_{p}}^{2} \prod_{j=1}^{r+1} n_{j}^{1-j} \delta_{p}^{j}, \\
& A=\sum_{p=1}^{r} \sum_{L_{p} \subset R} \sigma_{L_{p}} \prod_{j=1}^{r+1} n_{j}^{1-\delta_{L_{p}}^{j},}, \\
& A_{\left(I_{k}\right)}^{\left(J_{h}\right)}=\sum_{p=k}^{r-h} \sum_{\substack{L_{p} \supset I_{k} \\
L_{p} \in R-J_{h}}} \sigma_{L_{p}}^{2} \prod_{t=1}^{r+1} n_{t}^{1-\delta_{L_{p}}^{t} J_{h},},
\end{aligned}
$$




$$
\begin{aligned}
& B_{\left(I_{k}\right)}=A_{\left(I_{k}\right)}+\sigma_{e}^{2}, \\
& B=A+\sigma_{e}^{2}, \\
& \bar{X}_{t_{l_{1} \cdots t_{l \beta}}}=\sum_{t_{j_{1}}} \cdots \sum_{t_{j_{r-\beta}} \sum_{t_{r+1}} x_{t_{1} \cdot t_{r}} t_{r+1},}, \\
& \left(J_{r-\beta}=R-L_{\beta}\right)
\end{aligned}
$$

$$
\bar{X}=\sum_{t_{1}} \cdots \sum_{t_{r+1}} x_{t_{1} \cdots t_{r} t_{r+1}}
$$

$$
\bar{x}_{t_{t_{1} \cdots t_{t_{\beta}}}}=\frac{1}{\prod_{j=1}^{r+1} n_{j}^{1-\delta_{L_{\beta}}^{j}}} \bar{X}_{t_{t_{1} \cdots t_{l_{\beta}}}},
$$

$$
\bar{x}=\frac{1}{n_{1} \cdots n_{r+1}} \bar{X}
$$

$$
\begin{gathered}
u_{t_{1} \cdots t_{r} t_{r+1}}=x_{t_{1} \cdots t_{r} t_{r+1}}-\mu, \\
\bar{U}_{t_{l_{1} \cdots t_{\beta}}}=\sum_{t_{j_{1}}} \cdots \sum_{t_{j_{r-\beta}}} \sum_{t_{r+1}} u_{t_{1} \cdots t_{r} r_{r+1}}, \\
\left(J_{r-\beta}=R-L_{\beta}\right)
\end{gathered}
$$

$$
\bar{U}=\sum_{t_{1}} \cdots \sum_{t_{r+1}} u_{t_{1} \cdots t_{r} r^{t} r+1} \text {, }
$$

$$
\bar{u}_{t_{t_{1} \cdots t_{l_{\beta}}}}=\frac{1}{\prod_{j=1}^{r+1} n_{j}^{1-\delta_{L_{\beta}}^{j}}} \bar{U}_{t_{l_{1} \cdots t_{\beta}}},
$$

and

$$
\bar{u}=\frac{1}{n_{1} \cdots n_{r+1}} \bar{U}
$$

Lemma 4.1. $\left\{A_{\left(I_{k}\right)}\right\}$ (not including $A$ ) are functionally independent.

Proof. By the Definition 4.1 it is obvious that every $A_{\left(l_{k}\right)}$ is the linear form of a number of $\sigma_{L_{p}}^{2}$ 's. For any pair of members of $\left\{A_{\left(I_{k}\right)}\right\}$ the one includes at least one $\sigma_{L_{p}}^{2}$ not included in the other, which completes the proof.

From Lemma 4.1 obtain

Lemma 4.2. $\left\{B_{\left(I_{k}\right)}\right\}$ (not including $B$ ) are functionally independent.

Lemma 4. 3 .

$$
A_{\left(I_{k}\right)}^{\left(\cdot J_{h}\right)}=\frac{1}{n_{j_{h}}}\left[A_{\left(I_{k}\right)}^{\left(J_{h-1}\right)}-A_{\left(I_{k}, J_{h}\right)}^{\left(J_{l-1}\right)}\right] .
$$

\section{Proof.}


(4.19)

$$
\begin{aligned}
& A_{\left(I_{k}\right)}^{\left(J_{h}\right)}=\sum_{p=k}^{r-h} \sum_{\substack{L_{p} I_{k} I_{k} \\
L_{p}<R-J_{h}}} \sigma_{L_{t=1}^{2}}^{2} \prod_{t=1}^{r+1} n_{t}^{1-\delta_{L_{p}}^{t} J_{h}}
\end{aligned}
$$

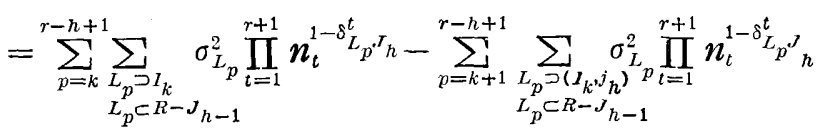

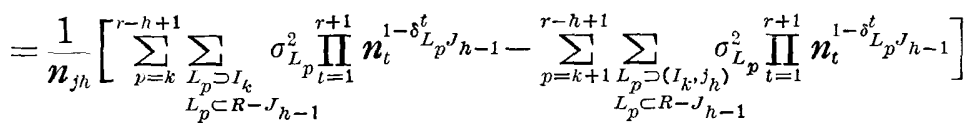

$$
\begin{aligned}
& =\frac{1}{n_{j h}}\left[A_{\left(I_{k}\right)}^{\left(J_{h-1}\right)}-A_{\left(I_{k}, j_{h}\right)}^{\left(J_{h-1}\right)}\right] .
\end{aligned}
$$

This lemma enables us to express $A_{\left(I_{k}\right)}^{\left(J_{h}\right)}$ in therms of $A_{\left(I_{k}, L_{p}\right)}$, which is given by

Lemma 4.4.

$$
A_{\left(I_{k}\right)}^{\left(J_{h}\right)}=\frac{1}{n_{j_{1}} \cdots n_{j_{h}}} \sum_{p=0}^{h} \sum_{L_{p} J_{h}}(-1)^{p} A_{\left(I_{k}, L_{p}\right)} .
$$

Proof. We shall give the proof by making use of the mathematical induction in $h$.

In case $h=1$, we have from Lemma 4.3

$$
\begin{aligned}
A_{\left(I_{k}\right)}^{\left(j_{1}\right)} & =\frac{1}{n_{j_{1}}}\left[A_{\left(I_{k}\right)}-A_{\left(I_{k}, j_{1}\right)}\right] \\
& =\frac{1}{n_{j_{1}}} \sum_{p=0}^{1} \sum_{L_{p} \sqsubset J_{1}}(-1)^{p} A_{\left(I_{k^{\prime}} L_{p}\right.} .
\end{aligned}
$$

Then assuming (4.20) to be valid in case $h=N$, i. e.,

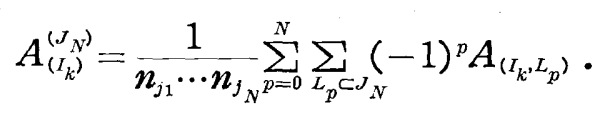

We shall prove this is also valid in case $h=N+1$ by using Lemma 4.3 and (4.22), which is given by

$$
\begin{aligned}
& \text { (4.23) } A_{\left(I_{k}\right)}^{\left(J_{N+1}\right)}=\frac{1}{n_{j_{N+1}}}\left[A_{\left(I_{k}\right)}^{\left(J_{N}\right)}-A_{\left(I_{k}, j_{N+1}\right.}^{\left(J_{N}\right)}\right] \\
& =\frac{1}{n_{j_{N+1}}}\left[\frac{1}{n_{j_{1}} \cdots n_{i_{N}}}\left\{\sum_{p=0}^{N} \sum_{L_{p} J_{N}}(-1)^{p} A_{\left(I_{k}, L_{p}\right)}-\sum_{p=0}^{N} \sum_{L_{p} J_{N}}(-1)^{p} A_{\left(I_{K^{\prime}} j_{N+1}, L_{p}\right)}\right\}\right]
\end{aligned}
$$

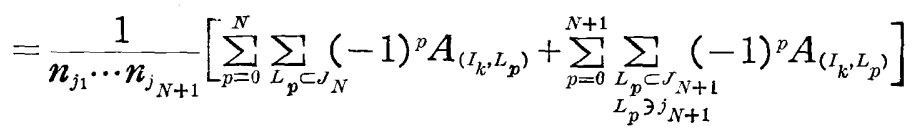




$$
=\frac{1}{n_{j_{1}} \cdots \bar{n}_{j_{N+1}}}-\sum_{p=0}^{N+1} \sum_{L_{p} \subset J_{N+1}}(-1)^{p} A_{\left(I_{k}, L_{p}\right)} .
$$

Now we observe the next lemma meaning the dependency between $A$ and $\left\{A_{\left(I_{k}\right)}\right\}$ or $B$ and $\left\{B_{\left(I_{k}\right)}\right\}$.

\section{Lemma 4.5.}

$$
\begin{aligned}
& A=\sum_{p=1}^{r} \sum_{L_{p} \subset R}(-1)^{p-1} A_{\left(L_{p}\right)}, \\
& B=\sum_{p=1}^{r} \sum_{L_{p} \subset R}(-1)^{p-1} B_{\left(L_{p}\right)} .
\end{aligned}
$$

Proof. By Definition 4.1 $A^{(R)}$ is the sum over the null index set and is equal to zero. On the other hand Lemma 4.4 should hold true even if $I_{k}$ is the null set, and we have

$$
\begin{aligned}
A^{(R)} & =\frac{1}{\prod_{j=1}^{r+1} n_{j}} \sum_{p=0}^{r} \sum_{L_{p} \subset R}(-1)^{p} A_{\left(L_{p}\right)} \\
& =\frac{1}{\prod_{j=1}^{r+1} n_{j}}\left[A+\sum_{p=1}^{r} \sum_{L_{p} \subset R}(-1)^{p} A_{(L p)}\right]=0 .
\end{aligned}
$$

This is equivalent to

$$
A=\sum_{p=1}^{r} \sum_{L_{p} \subset R}(-1)^{p-1} A_{\left(L_{p}\right)} .
$$

Here we need to prepare the following

Leema 4.6.

$$
\text { (4.2 8) } \sum_{t_{i_{1}}} \cdots \sum_{t_{i_{k}}} \sum_{\beta=0}^{k} \sum_{L_{\beta} \subset I_{k}}(-1)^{k-\beta} \bar{U}_{t_{l_{1} \cdots t_{l}}}^{2}=\sum_{t_{i_{1}}} \cdots \sum_{t_{i_{k}}}\left[\sum_{\beta=0}^{k} \sum_{L_{\beta} \subset I_{k}}(-1)^{k-\beta} U_{t_{l_{1}} \cdots t_{i_{\beta}}}\right]^{2} \text {. }
$$

Proof. Proof is given by making use of the mathematical induction. In case $k=1$, the proof is given by

$$
\begin{gathered}
\sum_{t_{i_{1}}}\left\{(-1) \bar{U}^{2}+\bar{U}_{t_{i_{1}}}^{2}\right\}=\sum_{t_{i_{1}}}\left(\bar{U}_{t_{i_{1}}}^{2}-\bar{U}^{2}\right) \\
=\sum_{t_{i_{1}}}\left(\bar{U}_{t_{i_{1}}}-\bar{U}\right)^{2} .
\end{gathered}
$$

Fnrther assuming (4.28) to be valid in case $k=N$, we have

$$
\text { (4.30) } \sum_{i_{i_{1}}, \cdots, t_{i_{N+1}}} \sum_{\beta=0}^{N+1} \sum_{L_{\beta} \in I_{N+1}}(-1)^{N+1-\beta} \bar{U}_{t_{l} \cdots l_{\beta}}^{2}
$$




$$
\begin{aligned}
& =\sum_{t_{i 1}, \cdots, t_{i+1}} \sum_{\beta=0}^{N} \sum_{L_{\beta} \subset I_{N}}\left[(-1)^{N-\beta} \bar{U}_{t_{l_{1}} \cdots t_{l_{\beta}} t_{t_{N}}}^{2}-(-1)^{N-\beta} \bar{U}_{t_{l_{1}} \cdots t_{l}}^{2}\right] \\
& \left.=\sum_{t_{i 1}, \cdots, t_{i_{N+1}}}\left[\left\{\sum_{\beta=0}^{N} \sum_{L_{\beta} \in I_{N}}(-1)^{N-\beta} \bar{U}_{t_{l_{1}} \cdots t_{L_{\beta}}}{ }_{i_{i}}\right\}^{2}\right\}^{2}-\left\{\sum_{\beta=0}^{N} \sum_{L_{\beta} \subset I_{N}}(-1)^{N-\beta} \bar{U}_{t_{t_{1}} \cdots t_{\beta}}\right\}^{2}\right]
\end{aligned}
$$

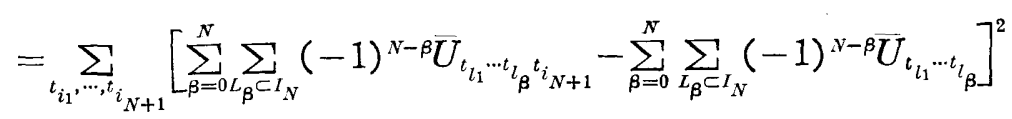

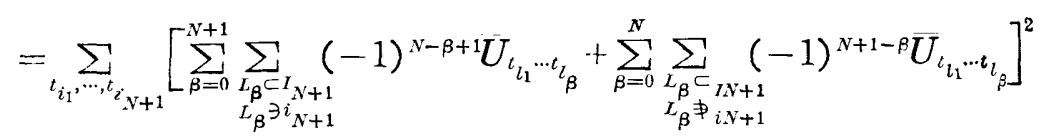

$$
\begin{aligned}
& =\sum_{i_{i}, \cdots, t_{i}}\left[\sum_{\beta=0}^{N+1} \sum_{L_{\beta} \subset t_{N+1}}(-1)^{N+1-\beta} \bar{U}_{t_{l_{1}} \cdots t_{\beta}}\right]^{2},
\end{aligned}
$$

which completes the proof of the lemma.

We shall derive the joint density function as the generalization of (3.9), which is enunciated in

Theorem 4.1. The joint density function of all $x_{t_{1 \cdots} t_{r+1}}$ 's is given by

$$
\begin{aligned}
f(\boldsymbol{x})= & (2 \pi)^{-n_{1} \cdots n_{r+1 / 2} B^{-1 / 2}} \prod_{k=1}^{r} \prod_{I_{k} \subset R}\left\{B_{\left(I_{k}\right)}\right\}^{-\left(n_{i_{1}}-1\right) \cdots\left(n_{i k}-1\right) / 2}\left(\sigma_{e}^{2}\right)^{-n_{1} \cdots n_{r}\left(n_{r+1}-1\right) / 2} \\
& \cdot \exp \left[-\frac{1}{2}\left\{\frac{\prod_{j=1}^{r+1} n_{j}(\bar{x}-\mu)^{2}}{B}+\sum_{k=1}^{r} \sum_{I_{k} \subset R} \frac{S_{\left(I_{k}\right)} B_{\left(I_{k}\right)}}{B}+\frac{S_{e}}{\sigma_{e}^{2}}\right\}\right]
\end{aligned}
$$

where

$$
\begin{aligned}
& S_{\left(I_{k}\right)}=\prod_{j=1}^{r+1} n_{j}^{1-\delta \delta_{t_{k}}^{j}} \sum_{t_{i_{1}}} \cdots \sum_{t_{i_{k}}}\left\{\sum_{\beta=0}^{k} \sum_{L_{\beta} \subset I_{k}}(-1)^{k-\beta} \bar{x}_{t_{l_{1}} \cdots t_{l_{\beta}}}\right\}^{2}, \\
& S_{e}=\sum_{t_{1}} \cdots \sum_{t_{r+1}}\left(x_{t_{1} \cdots t_{r} t_{r+1}}-\bar{x}_{t_{1} \cdots t_{r}}\right)^{2} .
\end{aligned}
$$

Proof. Let us at first transform the variance-covariance matrix given in (4.2) by the orthogonal matrix which is the Kronecker product of the matrices defined in (2.2), and we have

$$
\begin{aligned}
\left(\boldsymbol{T}_{n_{1}} \otimes \boldsymbol{T}_{n_{2}} \otimes \cdots \otimes \boldsymbol{T}_{n_{r+1}}\right) \boldsymbol{V}\left(\boldsymbol{T}_{n_{1}} \otimes \boldsymbol{T}_{n_{2}} \otimes \cdots \otimes \boldsymbol{T}_{n_{r+1}}\right)^{\prime} \\
=\sum_{k=1}^{r} \sum_{I_{k} \subset R} \sigma_{I_{k}}^{2} \prod_{j=1}^{r+1} n_{j}^{1-\delta J_{I_{k}}^{j}}\left[\prod_{t=1}^{r} \otimes\left(\boldsymbol{H}_{n_{t}}^{1-\delta_{J_{k}}^{t}} \boldsymbol{I}_{n_{t}}^{\delta_{I_{k}}^{t}}\right)\right] \otimes \boldsymbol{H}_{n_{r+1}} \\
\quad+\sigma_{e}^{2} \boldsymbol{I}_{n_{1}} \otimes \boldsymbol{I}_{n_{2}} \otimes \cdots \otimes \boldsymbol{I}_{n_{r+1}} \\
=\sum_{k=1}^{r} \sum_{I_{k} \subset R} \sigma_{I_{k}}^{2} \prod_{j=1}^{r+1} n_{j}^{1-\delta_{I_{k}}^{j}}\left[\prod_{t=1}^{r} \otimes\left(\boldsymbol{H}_{n_{t}}^{1-\delta_{J_{k}}^{t}}\left(\boldsymbol{H}_{n_{t}}+\boldsymbol{K}_{n_{t}}\right)^{\delta_{I_{k}}^{t}}\right)\right] \otimes \boldsymbol{H}_{n_{r+1}}
\end{aligned}
$$




$$
\begin{aligned}
& +\sigma_{e}^{2} \prod_{j=1}^{r+1} \otimes\left(\boldsymbol{H}_{n_{j}}+\boldsymbol{K}_{n_{j}}\right) \\
& =\sum_{k=1}^{r} \sum_{I_{k} \subset R} \sigma_{I_{k}}^{2} \prod_{j=1}^{r+1} n_{j}^{1-\delta_{I_{k}}^{j}}\left[\prod_{t=1}^{r} \otimes\left(\boldsymbol{H}_{n_{t}}+\delta_{I_{k}}^{t} \boldsymbol{K}_{n_{t}}\right)\right] \otimes \boldsymbol{H}_{n_{\gamma+1}} \\
& +\sigma_{e}^{2} \prod_{j=1}^{r+1} \otimes\left(\boldsymbol{H}_{n_{j}}+\boldsymbol{K}_{n_{j}}\right) \\
& =\left[\sum_{p=1}^{r} \sum_{L_{p} \in R} \sigma_{L_{p}}^{2} \prod_{j=1}^{r+1} n_{j}^{1-\delta_{L_{p}}^{3}}+\sigma_{e}^{2}\right] \boldsymbol{H}_{n_{1}} \otimes \cdots \otimes \boldsymbol{H}_{n_{r}} \otimes \boldsymbol{H}_{n_{r+1}}
\end{aligned}
$$

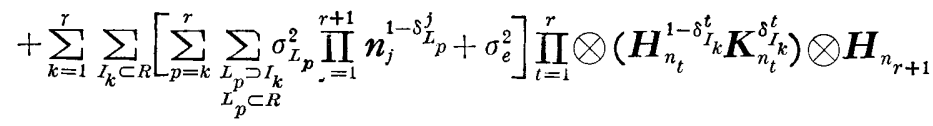

$$
\begin{aligned}
& +\sigma_{e}^{2} \boldsymbol{I}_{n_{1}} \otimes \cdots \otimes \boldsymbol{I}_{n_{r}} \otimes \boldsymbol{K}_{n_{r+1}} \\
& =B \prod_{j=1}^{r+1} \otimes \boldsymbol{H}_{n_{j}}+\sum_{k=1}^{r} \sum_{j_{k} \subset R} B_{\left(I_{k}\right)} \prod_{t=1}^{r} \otimes\left(\boldsymbol{H}_{n_{t}}^{1-\delta_{I_{k}}^{t}} \boldsymbol{K}_{n_{t}}^{\delta_{I_{k}}^{t}}\right) \otimes \boldsymbol{H}_{n_{r+1}} \\
& +\sigma_{e}^{2} \boldsymbol{I}_{n_{1}} \otimes \cdots \otimes \boldsymbol{I}_{n_{r}} \otimes \boldsymbol{K}_{n_{r+1}} .
\end{aligned}
$$

Thus the matrix (4.32) is expressed as the linear form of a number of matrices of the type

where

$$
\boldsymbol{A}_{n_{1}} \otimes \boldsymbol{A}_{n_{2}} \otimes \cdots \otimes \boldsymbol{A}_{n_{r}} \otimes \boldsymbol{H}_{n_{r+1}}
$$

$$
\boldsymbol{A}_{i}=\boldsymbol{H}_{i} \text { or } \boldsymbol{K}_{\boldsymbol{i}}
$$

and the matrix $\boldsymbol{I}_{n_{1}} \otimes \cdots \otimes \boldsymbol{I}_{n_{r}} \otimes \boldsymbol{K}_{n_{r+1}}$ and all of them are diagonal matrices with the diagonal elements eqnal to zero or one, and have no non-zero element in common. This fact leads us to the evaluation of the inverse of (4.2) as follows,

$$
\text { (4.33) } \begin{aligned}
& {\left[\left(\boldsymbol{T}_{n_{1}} \otimes \boldsymbol{T}_{n_{2}} \otimes \cdots \otimes \boldsymbol{T}_{n_{r+1}}\right) \boldsymbol{V}\left(\boldsymbol{T}_{n_{1}} \otimes \boldsymbol{T}_{n_{2}} \otimes \cdots \otimes \boldsymbol{T}_{n_{r+1}}\right)^{\prime}\right]^{-1} } \\
&=\frac{1}{B} \prod_{j=1}^{r+1} \otimes \boldsymbol{H}_{n_{j}}+\sum_{k=1}^{r} \sum_{I_{k} \in R} \frac{1}{B_{\left(I_{k}\right)}} \prod_{t=1}^{r} \otimes\left(\boldsymbol{H}_{n_{t}}^{1-\delta_{I_{k}}^{t}} \boldsymbol{K}_{n_{t}}^{\delta_{\boldsymbol{I}_{k}}^{t}}\right) \otimes \boldsymbol{H}_{n_{r+1}} \\
&+\frac{1}{\sigma_{e}^{2}} \boldsymbol{I}_{n_{1}} \otimes \cdots \otimes \boldsymbol{I}_{n_{r}} \otimes \boldsymbol{K}_{n_{r+1}} \\
&= \frac{1}{B} \prod_{j=1}^{r+1} \otimes \boldsymbol{H}_{n_{j}}+\sum_{k=1}^{r} \sum_{I_{k} \in R} \frac{1}{\boldsymbol{B}_{\left(I_{k}\right)}} \prod_{t=1}^{r} \otimes\left\{\boldsymbol{H}_{n_{t}}^{1-\delta_{I_{k}}^{t}}\left(\boldsymbol{I}_{n_{t}}-\boldsymbol{H}_{n_{t}}\right)^{\delta_{I_{k}}^{t}}\right\} \otimes \boldsymbol{H}_{n_{r+1}} \\
&+\frac{1}{\sigma_{e}^{2}} \boldsymbol{I}_{n_{1}} \otimes \cdots \otimes \boldsymbol{I}_{n_{r}} \otimes\left(\boldsymbol{I}_{n_{r+1}}-\boldsymbol{H}_{n_{r+1}}\right) .
\end{aligned}
$$

Therefore, in view of (2.3), we have 


$$
\begin{aligned}
& \text { (4. 34) } \quad \boldsymbol{V}^{-1}=\frac{1}{\prod_{j=1}^{r+1} n_{j} B} \boldsymbol{E}_{n_{1}} \otimes \cdots \otimes \boldsymbol{E}_{n_{r}} \otimes \boldsymbol{E}_{n_{r+1}} \\
& +\sum_{k=1}^{r} \sum_{I_{k} \subset R} \frac{1}{B_{\left(I_{k}\right)}} \prod_{t=1}^{r} \otimes\left\{\left(\frac{1}{\boldsymbol{n}_{t}} \boldsymbol{E}_{n_{t}}\right)^{1-\delta_{I_{k}}^{t}}\left(\boldsymbol{I}_{n_{t}}-\frac{1}{\boldsymbol{n}_{t}} \boldsymbol{E}_{n_{t}}\right)^{\delta_{I_{k}}^{t}}\right\} \otimes \frac{1}{\boldsymbol{n}_{r+1}} \boldsymbol{E}_{n_{r+1}} \\
& +\frac{1}{\sigma_{e}^{2}} \boldsymbol{I}_{n_{1}} \otimes \cdots \otimes \boldsymbol{I}_{n_{r}} \otimes\left(\boldsymbol{I}_{n_{r+1}}-\frac{1}{n_{r+1}} \boldsymbol{E}_{n_{r+1}}\right) \\
& =\frac{1}{\prod_{j=1}^{r+1} n_{j} B} \boldsymbol{E}_{n_{1}} \otimes \cdots \otimes \boldsymbol{E}_{n_{r}} \otimes \boldsymbol{E}_{n_{r+1}} \\
& +\sum_{k=1}^{r} \sum_{I_{k} \subset R} \frac{1}{B_{\left(I_{k}\right)}}\left[\sum_{\beta=0}^{k} \sum_{L_{\beta} \subset I_{k}}(-1)^{k-\beta} \prod_{t=1}^{r} \otimes\left(\boldsymbol{H}_{n_{t}}^{1-\delta_{L_{\beta}}^{t}} \boldsymbol{I}_{n_{t}}^{\delta_{L_{\beta}}^{t}}\right) \otimes \boldsymbol{E}_{n_{r+1}} \prod_{j=1}^{r+1}\left(\frac{1}{n_{j}}\right)^{1-\delta_{L_{\beta}}^{j}}\right] \\
& +\frac{1}{\sigma_{e}^{2}} \boldsymbol{I}_{n_{1}} \otimes \cdots \otimes \boldsymbol{I}_{n_{r}} \otimes\left(\boldsymbol{I}_{n_{r}}-\frac{1}{\boldsymbol{n}_{r+1}} \boldsymbol{E}_{n_{r+1}}\right) \text {. }
\end{aligned}
$$

Let $\boldsymbol{U}$ be the column vector of $u_{t_{1}} \cdots t_{r+1}$, then we have by using Lemma 4.6

$$
\begin{aligned}
& \boldsymbol{U}^{\prime} \prod_{j=1}^{\frac{1}{r+1} n_{j}} \boldsymbol{E}_{n_{1}} \otimes \cdots \otimes \boldsymbol{E}_{n_{r}} \otimes \boldsymbol{E}_{n_{r+1}} \boldsymbol{U} \\
& =\frac{1}{\prod_{j=1}^{r+1} n_{j}} \overline{\boldsymbol{U}}^{2} \\
& =\prod_{j=1}^{r+1} n_{j}(\bar{x}-\mu)^{2}
\end{aligned}
$$

(4. 36) $\quad \boldsymbol{U}^{\prime}\left[\sum_{\beta=0}^{k} \sum_{L_{\beta} \subset I_{k}}(-1)^{k-\beta} \prod_{t=1}^{r} \otimes\left(\boldsymbol{E}_{n_{t}}^{1-\delta_{L_{\beta}}^{t}} \boldsymbol{I}_{n_{t}}^{\delta_{L_{\beta}}^{t}}\right) \otimes \boldsymbol{E}_{n_{r+1}} \prod_{j=1}^{r+1}\left(\frac{1}{n_{j}}\right)^{1-\delta_{L_{\beta}}^{j}}\right] \boldsymbol{U}$

$$
\begin{aligned}
& =\sum_{\beta=0}^{k} \sum_{L_{\beta} \subset I_{k}}(-1)^{k-\beta} \sum_{t_{l_{1}}} \cdots \sum_{t_{l_{\beta}}} \overline{\boldsymbol{U}}_{{ }_{t} \cdots t_{1}}^{2} \prod_{\beta} \prod_{j=1}^{i+1}\left(\frac{1}{n_{j}}\right)^{1-\delta_{L_{\beta}}^{j}} \\
& =\sum_{\beta=0}^{k} \sum_{L_{\beta} \subset I_{k}}(-1)^{k-\beta} \prod_{j=1}^{r+1} n_{j}^{1-\delta_{L_{\beta}}^{j}} \sum_{t_{l_{1}}} \cdots \sum_{{ }_{t_{\beta}}} \bar{u}_{t_{l_{1}} \cdots t_{t_{\beta}}}^{2} \\
& =\sum_{t_{1}} \cdots \sum_{t_{r+1}}\left[\sum_{\beta=0}^{k} \sum_{L_{\beta} \subset I_{k}}(-1)^{k-\beta} \bar{u}_{t_{1} \cdots t_{\beta}}^{2}\right] \\
& =\prod_{j=1}^{r+1} n_{j}^{1-\delta_{I_{k}}^{j}} \sum_{t_{i_{1}}} \cdots \sum_{t_{i_{k}}}\left[\sum_{\beta=0}^{k} \sum_{L_{\beta} \subset I_{k}}(-1)^{k-\beta} \bar{u}_{t_{l_{1}} \cdots t_{l_{\beta}}}^{2}\right] \\
& =\prod_{j=1}^{r+1} \boldsymbol{n}_{j}^{1-\delta^{j}{ }_{k}} \sum_{t_{i_{j}}} \cdots \sum_{t_{i_{k}}}\left[\sum_{\beta=0}^{k} \sum_{L_{\beta} \in I_{k}}(-1)^{k-\beta} \overline{\boldsymbol{u}}_{t_{l_{1}} \cdots t_{l \beta}}\right]^{2} \text {. }
\end{aligned}
$$




$$
\begin{aligned}
& =\prod_{j=1}^{r+1} n_{j}^{1-\delta_{I_{k}}^{j}} \sum_{t_{i_{1}}} \cdots \sum_{t_{i_{k}}}\left[\sum_{\beta=0}^{k} \sum_{L_{\beta} \in I_{k}}(-1)^{k-\beta}\left(\bar{x}_{t_{l_{1}} \cdots t_{l}}-\mu\right)\right]^{2} \\
& =\prod_{j=1}^{r+1} n_{j}^{1-\delta_{l_{k}}^{j}} \sum_{t_{i_{1}}} \cdots \sum_{t_{i_{k}}}\left[\sum_{\beta=0}^{k} \sum_{L_{\beta} \in I_{k}}(-1)^{k-\beta} \bar{x}_{t_{l_{1}} \cdots t_{l_{B}}}\right]^{2} \\
& =S_{\left(I_{k}\right)},
\end{aligned}
$$

and

$$
\text { (4.37) } \begin{aligned}
\boldsymbol{U}^{\prime} \boldsymbol{I}_{n_{1}} \otimes \cdots \otimes \boldsymbol{I}_{n_{r}} \otimes\left(\boldsymbol{I}_{n_{r+1}}-\frac{1}{n_{r+1}} \boldsymbol{E}_{n_{r+1}}\right) \boldsymbol{U} \\
=\boldsymbol{U}^{\prime} \boldsymbol{I}_{n_{1}} \otimes \cdots \otimes \boldsymbol{I}_{n_{r+1}} \boldsymbol{U}-\frac{1}{n_{r+1}} \boldsymbol{U}^{\prime} \boldsymbol{I}_{n_{1}} \otimes \cdots \otimes \boldsymbol{I}_{n_{r}} \otimes \boldsymbol{E}_{n_{r+1}} \boldsymbol{U} \\
=\sum_{t_{1}} \cdots \sum_{t_{r+1}} u_{t_{1} \cdots t_{r} t_{r+1}}^{2}-\frac{1}{n_{r+1}} \sum_{t_{1}} \cdots \sum_{t_{r}} \bar{U}_{t_{1} \cdots t_{r}}^{2} \\
=\sum_{t_{1}} \cdots \sum_{t_{r+1}}\left[u_{t_{1} \cdots t_{r} t_{r+1}}^{2}-\bar{u}_{t_{1} \cdots t_{r}}^{2}\right] \\
=\sum_{t_{1}} \cdots \sum_{t_{r+1}}\left[u_{t_{1} \cdots t_{r} t_{r+1}}-\bar{u}_{t_{1} \cdots t_{r}}\right]^{2} \\
=\sum_{t_{1}} \cdots \sum_{t_{r+1}}\left[\boldsymbol{x}_{t_{1} \cdots t_{r} t_{r+1}}-\overline{\boldsymbol{x}}_{t_{1} \cdots t_{r}}\right]^{2} \\
=S_{e} .
\end{aligned}
$$

The quadratic form in the exponent of our density function is then seen to be

$$
\boldsymbol{U}^{\prime} \boldsymbol{V}^{-1} \boldsymbol{U}=\frac{\prod_{j=1}^{r+1} n_{j}(\bar{x}-\mu)^{2}}{B}+\sum_{k=1}^{r} \sum_{I_{k} \in R} \frac{S_{\left(I_{k}\right)}}{B_{\left(I_{k}\right)}}+\frac{S_{e}}{\sigma_{e}^{2}} .
$$

On the other hand we obtain easily from (4.33)

$$
|\boldsymbol{V}|=B \prod_{k=1}^{r} \prod_{I_{k} \in R}\left\{\boldsymbol{B}_{\left(I_{k}\right)}\right\}^{\left(n_{i_{1}}-1\right) \cdots\left(n_{i_{k}}-1\right)}\left\{\sigma_{e}^{2}\right\}^{n_{1} \cdots n_{r^{\prime}}\left(n_{r+1}-1\right)}
$$

At last we have (4.31) by (4.38) and (4.39), which completes the proof.

Theorem 4.2. $S_{\left(I_{k}\right)} / B_{\left(I_{k}\right)}$ is distributed in central chi-square distribution with $\left(n_{i_{1}}-1\right) \cdots\left(n_{i_{k}}-1\right)$ degrees of freedom for every $I_{k} \subset R, S_{e} / \sigma_{e}^{2}$ with $n_{1} \cdots n_{r}\left(n_{r+1}-1\right)$ degrees of freedom also, and these all are distributed independently to each other.

\section{Proof.}

Let $\boldsymbol{X}$ be a column vector of observations, $\mu$ be a column vector $(\mu, \mu$, $\cdots, \mu)^{\prime}$.

And let $\boldsymbol{T}_{n_{t}}^{(H)}, \boldsymbol{T}_{n_{t}}^{(K)}$ be matrices, modified $\boldsymbol{T}_{n_{t}}$ respectively, as follows; 


$$
\boldsymbol{T}_{n_{t}}^{(H)}=\left(\begin{array}{ccc}
\frac{1}{\sqrt{n_{t}}} & \cdots \cdots & \frac{1}{V} \\
0 & \cdots \cdots & 0 \\
\vdots & & \vdots \\
0 & \cdots \cdots & 0
\end{array}\right),
$$

$$
\boldsymbol{T}_{n_{t}}^{(K)}=\boldsymbol{T}_{n_{t}}-\boldsymbol{T}_{n_{t}}^{(I I)}
$$

Then we have another expression of $\boldsymbol{U}^{\prime} \boldsymbol{V}^{-1} \boldsymbol{U}$ given by

(4.42) $\boldsymbol{U}^{\prime} \boldsymbol{V}^{-1} \boldsymbol{U}$

$$
\begin{aligned}
& \left.=\boldsymbol{U}^{\prime}\left(\boldsymbol{T}_{n_{1}} \otimes \cdots \otimes \boldsymbol{T}_{n_{r+1}}\right)^{\prime}\left[\boldsymbol{T}_{n_{1}} \otimes \cdots \otimes \boldsymbol{T}_{n_{r+1}}\right) \boldsymbol{V}\left(\boldsymbol{T}_{n_{1}} \otimes \cdots \otimes \boldsymbol{T}_{n_{r+1}}\right)^{\prime}\right]^{-1} \\
& \left(\boldsymbol{T}_{n_{1}} \otimes \cdots \otimes \boldsymbol{T}_{n_{r+1}}\right) \boldsymbol{U} \\
& =\boldsymbol{U}^{\prime}\left(\boldsymbol{T}_{n_{1}} \otimes \cdots \otimes \boldsymbol{T}_{n_{r+1}}\right)^{\prime} \\
& \cdot\left[\frac{1}{B} \boldsymbol{H}_{n_{1}} \otimes \cdots \otimes \boldsymbol{H}_{n_{r+1}}+\sum_{k=1}^{r} \sum_{I_{k} \subset R} \frac{1}{\boldsymbol{B}_{\left(I_{k}\right)}} \prod_{l=1}^{r} \otimes\left(\boldsymbol{H}_{n_{t}}^{1-\delta_{n_{t}}^{t}} \boldsymbol{K}_{n_{t}}^{\delta_{I_{k}}^{t}}\right) \otimes \boldsymbol{H}_{n_{r+1}}\right. \\
& \left.+\frac{1}{\sigma_{e}^{2}} \boldsymbol{I}_{n 1} \otimes \cdots \otimes \boldsymbol{I}_{n_{r}} \otimes \boldsymbol{K}_{n_{r+1}}\right] \\
& \cdot\left(\boldsymbol{T}_{n_{1}} \otimes \cdots \otimes \boldsymbol{T}_{n_{r+1}}\right) \boldsymbol{U} \\
& =\frac{1}{B}(\boldsymbol{X}-\boldsymbol{\mu})^{\prime}\left(\boldsymbol{T}_{n_{1}}^{(H)} \otimes \cdots \otimes \boldsymbol{T}_{n_{r+1}}^{(H)}\right)^{\prime}\left(\boldsymbol{T}_{n_{1}}^{(H)} \otimes \cdots \otimes \boldsymbol{T}_{n_{r+1}}^{(H)}\right)(\boldsymbol{X}-\boldsymbol{\mu}) \\
& +\sum_{k=1}^{r} \sum_{I_{k} \subset R} \frac{1}{B_{\left(I_{k}\right)}} \boldsymbol{X}^{\prime}\left[\prod_{t=1}^{r} \otimes\left(\boldsymbol{T}_{n_{t}}^{(H)^{1-\delta_{I}}}{ }_{I_{k}} \boldsymbol{T}_{n_{t}}^{(k)}{ }_{I_{k}}^{t}\right) \otimes \boldsymbol{T}_{n_{r+1}}^{(H)}\right]^{\prime} \\
& \cdot\left[\prod_{t=1}^{r} \otimes\left(\boldsymbol{T}_{n_{t}}^{(H)}{ }^{1-\delta_{I_{k}}^{t}} \boldsymbol{T}_{n_{t}}^{(K)^{\delta}}{ }_{I_{k}}^{t}\right) \otimes \boldsymbol{T}_{n_{r+1}}^{(H)}\right] \boldsymbol{X} \\
& +\frac{1}{\sigma_{e}^{2}} \boldsymbol{X}^{\prime}\left(\boldsymbol{T}_{n_{1}} \otimes \cdots \otimes \boldsymbol{T}_{n_{r}} \otimes \boldsymbol{T}_{n_{r+1}}^{(k)}\right)^{\prime}\left(\boldsymbol{T}_{n_{1}} \otimes \cdots \otimes \boldsymbol{T}_{n_{r}} \otimes \boldsymbol{T}_{n_{r+}}^{(K)}\right) \boldsymbol{X} \\
& =\frac{1}{B}(X-\mu)^{\prime} Q_{0}^{\prime} Q_{0}(X-\mu) \\
& \sum_{k=1}^{r} \sum_{I_{k} \subset R} \frac{1}{B_{\left(I_{k}\right)}} \boldsymbol{X}^{\prime} \boldsymbol{Q}_{\left(I_{k}\right)}^{\prime} \boldsymbol{Q}_{\left(I_{k}\right)} \boldsymbol{X}+\frac{1}{\sigma_{e}^{2}} \boldsymbol{X}^{\prime} \boldsymbol{Q}_{e}^{\prime} \boldsymbol{Q}_{e} \boldsymbol{X},
\end{aligned}
$$

where

$$
\begin{aligned}
& \boldsymbol{Q}_{0}=\boldsymbol{T}_{n_{1}}^{(H)} \otimes \cdots \otimes \boldsymbol{T}_{n_{r+1}}^{(H)}, \\
& \boldsymbol{Q}_{\left(I_{k}\right)}=\prod_{t=1}^{r} \otimes\left(\boldsymbol{T}_{n_{t}}^{(H)}{ }^{1-\delta_{I_{k}}^{t}} \boldsymbol{T}_{n_{t}}^{\left.(K)^{\delta}\right)^{t}}\right) \otimes \boldsymbol{T}_{n_{r+1}}^{(H)}, \quad I_{k} \subset R ; k=1,2, \cdots, r,
\end{aligned}
$$

and 


$$
\boldsymbol{Q}_{e}=\boldsymbol{T}_{n_{1}} \otimes \cdots \otimes \boldsymbol{T}_{n_{r}} \otimes \boldsymbol{T}_{n_{r+1}}^{(K)}
$$

Here the following results are easily obtained.

(A) For $I_{k} \subset R, Q^{\prime}{ }_{\left(I_{k}\right)} \boldsymbol{Q}_{\left(I_{k}\right)} \boldsymbol{V} / B_{\left(I_{k}\right)}$ and $\boldsymbol{Q}_{e}^{\prime} \boldsymbol{Q}_{e} \boldsymbol{V} / \sigma_{e}^{2}$ are all idempotents since it holds that

$$
\begin{aligned}
& \boldsymbol{Q}_{\left(I_{k}\right)}^{\prime}\left(\boldsymbol{Q}_{\left(I_{k}\right)} \boldsymbol{V} \boldsymbol{Q}_{\left(I_{k}\right)}^{\prime}\right) \boldsymbol{Q}_{\left(I_{k}\right)} \boldsymbol{V} / B_{\left(I_{k}\right)}^{2}=Q_{\left(I_{k}\right)}^{\prime} B_{\left(I_{k}\right)} \boldsymbol{I} \boldsymbol{Q}_{\left(I_{k}\right)} \boldsymbol{V} / B_{\left(I_{k}\right)}^{2} \\
& \quad=Q_{\left(I_{k}\right)}^{\prime} \boldsymbol{Q}_{\left(I_{k}\right)} \boldsymbol{V} / B_{\left(I_{k}\right)}, \\
& \boldsymbol{Q}_{e}^{\prime}\left(\boldsymbol{Q}_{e} \boldsymbol{V} \boldsymbol{Q}_{e}^{\prime}\right) \boldsymbol{Q}_{e} \boldsymbol{V} /\left(\sigma_{e}^{2}\right)^{2}=\boldsymbol{Q}_{e}^{\prime} \sigma_{e}^{2} \boldsymbol{I} \boldsymbol{Q}_{e} \boldsymbol{V} /\left(\sigma_{e}^{2}\right)^{2} \\
& \quad=\boldsymbol{Q}_{e}^{\prime} \boldsymbol{Q}_{e} \boldsymbol{V} / \sigma_{e}^{2}
\end{aligned}
$$

(B) $\varepsilon\left[\boldsymbol{Q}_{\left(I_{k}\right)} \boldsymbol{X}\right]=\boldsymbol{Q}_{\left(I_{k}\right)} \boldsymbol{\mu}=\boldsymbol{O}$ for $I_{k} \subset R ; k=1,2, \cdots, \boldsymbol{r}$,

$$
\varepsilon\left[\boldsymbol{Q}_{e} \boldsymbol{X}\right]=\boldsymbol{Q}_{e} \boldsymbol{\mu}=\boldsymbol{O} \text {, }
$$$$
\varepsilon\left[\boldsymbol{X}^{\prime} \boldsymbol{Q}_{\left(I_{k}\right)}^{\prime} \boldsymbol{Q}_{\left(I_{k}\right)} \boldsymbol{X} /\left(\boldsymbol{n}_{i_{1}}-1\right) \cdots\left(\boldsymbol{n}_{i_{k}}-1\right)\right]=\boldsymbol{B}_{\left(I_{k}\right)} \text { for } I_{k} \subset R ; k=1,2, \cdots, \boldsymbol{r} \text {, }
$$$$
\varepsilon\left[X^{\prime} Q_{e}^{\prime} Q_{e} X / n_{1} \cdots n_{r}\left(n_{r+1}-1\right)\right]=\sigma_{e}^{2} \text {. }
$$

(C) $\operatorname{rank}\left[\boldsymbol{Q}_{\left(I_{k}\right)}^{\prime} \boldsymbol{Q}_{\left(I_{k}\right)} \boldsymbol{V}\right]=\left(\boldsymbol{n}_{i_{1}}-1\right) \cdots\left(n_{i_{k}}-1\right)$ for $I_{k} \subset R ; k=1,2, \cdots, r$, $\operatorname{rank}\left[Q_{e}^{\prime} Q_{e} V\right]=n_{1} n_{2} \cdots n_{r}\left(n_{r+1}-1\right)$.

(D) $\boldsymbol{Q}_{\left(I_{k}\right)} \boldsymbol{V} \boldsymbol{Q}_{\left(I_{k}\right)}^{\prime} \boldsymbol{O}$ for $I_{k} \neq J_{k}$.

This consideration leads us to the proof of the theorem.

Now, in our model, the random variable is $\boldsymbol{X}=X_{11 \cdots 1}, \cdots, X_{11 \cdots n} ; X_{21 \cdots 1}$, $\left.\cdots, X_{21 \cdots n_{r+1}} ; \cdots ; X_{n_{1} \cdots n_{r}}, \cdots, X_{n_{1} \cdots n_{r^{n} n+1}}\right)$, the sample space $R^{X}$ is a $n_{1} n_{2} \cdots n_{r+1^{-}}$ dimensional Euclidean space, and the family $\mathfrak{B}^{x}$ is specified by the parameter $\theta=\left(\mu, \sigma_{I_{k}}^{2}, \sigma_{e}^{2} ; I_{k} \subset R, k=1,2, \cdots, r\right)$, whose space is of $\left(2^{r}+1\right)$-dimension, wher $-\infty<\mu<+\infty, 0 \leqq \sigma_{I_{k}}^{2}<+\infty$ and $0 \leqq \sigma_{e}^{2}<+\infty$.

We shall consider the transformations of the original parameters and statistics such that

$$
\tau^{(1)}=\prod_{j=1}^{r+1} n_{j} \frac{\mu}{B},
$$

$$
\tau_{\left(I_{k}\right)}^{(2)}=-\frac{1}{2 B_{\left(I_{k}\right)}}, \quad I_{k} \subset R, k=1,2, \cdots, r,
$$

$$
\tau^{(3)}=-\frac{1}{2 \sigma_{e}^{2}},
$$

$$
Z^{(1)}=\bar{x},
$$

$$
Z_{\left(I_{k}\right)}^{(2)}=S_{\left(I_{k}\right)}, \quad I_{k} \subset R ; k=1,2, \cdots, r .
$$

$$
Z^{(3)}=S_{e} \text {. }
$$


Since we observe the independency of the class of parametric functions $\left\{B_{\left(I_{k}\right)} ; I_{k} \subset R, k=1,2, \cdots, r\right\}$ in Lemma 4.2 , it is seen that the transformation (4.43), $\cdots$, (4.45) from $\theta$ to $\tau=\left(\tau^{(1)}, \tau_{\left(I_{k}\right)}^{(2)}, \tau^{(3)} ; I_{k} \subset R, k=1,2, \cdots, r\right)$ is one-toone. Therefore we can say that $\mathfrak{B}^{x}$ is specified by $\tau$, where $-\infty<\tau^{(3)} \leqq \tau_{(M)}^{(2)}$ $<\tau_{(N)}^{(2)} \leqq 0$ for any pair $(M, N)$ such as $M \supset N, M \subset R, N \subset R$. Further we should notice that $B$ is a function of $B_{\left(I_{k}\right)}$ 's as seen in Lemma 4.5 , and $Z^{(1)}$, $Z_{\left(I_{k}\right)}^{(2)}$ 's and $Z^{(3)}$ are functionally independent to each other where the proof is omitted.

Then, under the new parameter $\tau$, by using the above-mentioned results we obtain the probability density function of $\boldsymbol{X}$ as follows;

$$
K_{(\cdot)} \exp \left[\tau^{(1)} Z^{(1)}+\sum_{k=1}^{r} \sum_{I_{k} \subset R} \tau_{\left(I_{k}\right)}^{(2)} Z_{\left(I_{k}\right)}^{(2)}+\tau^{(3)} Z^{(3)}+g\left(\tau_{\left(I_{k}\right)}^{(2)} \mid I_{k} \subset R\right) h\left(Z^{(1)}\right)\right]
$$

Hence the sufficient statistic for $\mathfrak{B}^{x}$ is $Z=\left(Z^{(1)}, Z_{I_{k}}^{(2)}, Z^{(3)} ; I_{k} \subset R, k=1,2, \cdots, r\right)$. In order to show $\mathfrak{B}^{z}$ in this type be complete, we need to use Gautschi's lemma or the following generalized lemma.

Let $Y^{(k)}$ be a $k$-dimensional Euclidean space with the point $y^{(k)}=\left(y_{1}, y_{2}\right.$, $\left.\cdots, y_{k}\right)$. We shall write the first $j$ components as $y^{(j)}$ and the remaining components $y^{((j))}$ so that we write $y^{(k)}$ in tha following different fashion $y^{(k)}=\left(y^{(j)}, y^{((j))}\right)=\left(y^{(k-1)}, y_{k}\right)$, etc. And let $\tau^{(k)}=\left(\tau_{1}, \tau_{2}, \cdots, \tau_{k}\right)$ be the point of a $k$-dimensional Euclidean space, then the notation $\tau^{(j)}$ is to be understood in the way above stated.

Lemma 4. 7. Let

$$
\mathfrak{B}^{Y^{(k)}}=\left\{P_{\mathrm{r}^{(k)}}^{Y^{(k)}} \mid \tau^{(k)} \in \omega\right\},
$$

where $\omega$ is a Borel set in an Euclidean space containing a non-degenerate $k$-dimensional interval, be the family of measures $P_{\tau(k)}^{r(k)}$ on the additive family of subset in the space of point $y^{(k)}$, having the density

(4.50) $\quad p_{\tau^{(k)}}\left(y^{(k)}\right)=C\left(\tau^{(k)}\right) h\left(y^{(k)}\right) \exp \left[\sum_{i=1}^{k} \tau_{i} y_{i}+g\left(\tau^{(s)}, y^{(s))}\right)\right]$

with respect to Lebesgue measure. Then $\mathfrak{B}^{Y^{(k)}}$ is strongly complete.

The proof of this was explained in [2].

In the estimation problem of the parameters, the estimates usually adopted in the practice of statistical inferences are unbiased and based on the sufficient statistic $Z$ defined above. As we can observe our family $\mathfrak{B}^{Z}$ is complete by making use of Lemma 4.7 , we have

Theorem 4.3. In our random effect model, the usual estimates of the parameters, such as the general mean and the variance components of random treatment effects, are the best unbiased (unique) estimates among 


\section{all unbiased estimates.}

\section{$\S 5$. Nested design.}

We shall treat the problem of the estimation in the random effect model for the nested design, whose model equation is given by

$$
\begin{aligned}
x_{t_{1} \cdots t_{r} t_{r+1}}=\mu+\sum_{A \subset R} a\left(1,2, \cdots, A ; t_{1},\right. & \left.t_{2}, \cdots, t_{a}\right)+e_{t_{1} \cdots t_{r} t_{r+1}}, \\
& \left(t_{j}=1,2, \cdots, n_{j} ; j=1,2, \cdots, r+1\right),
\end{aligned}
$$

where $\mu$ denotes the general mean, $a\left(1 ; t_{1}\right)$ is the effect of the lst factor labeled $t_{1}, a\left(1,2, \cdots, A ; t_{1}, t_{2}, \cdots, t_{a}\right)$ is the effect of the $a$-th factor labeled $\left(t_{1}, \cdots, t_{a}\right)$ within the nested plot labeled $\left(t_{1}, \cdots, t_{a-1}\right)$ for the 1st, 2 nd, $\cdots$, $(a-1)$-th factors, and $e_{t_{1} \cdots t_{r} t_{r+1}}$ is the error term.

We assume that $\mu$ is a constant, all $a\left(1,2, \cdots, A ; t_{1}, t_{2}, \cdots, t_{a}\right)$ 's and $e_{t_{1} \cdots t_{r} t_{r+1}}$ 's are distributed independently to each other as normal with mean all equal to zero and the variance of $a\left(1,2, \cdots, A ; t_{1}, t_{2}, \cdots, t_{a}\right)$ all equal to $\sigma_{A}^{2}$, the variance of $e_{t_{1} \cdots t_{r} t_{1+r}}$ all equal to $\sigma_{e \bullet}^{2}$

The variance-covariance matrix of all observation is given by

$$
\boldsymbol{V}=\sum_{A=1}^{R} \sigma_{A}^{2}\left[\prod_{j=1}^{r} \otimes\left(\boldsymbol{E}_{n_{j}}^{1-\delta_{A}^{j}} \boldsymbol{I}_{n_{j}}^{\mathcal{S}}\right)\right] \otimes \boldsymbol{E}_{n_{r+1}}+\sigma_{e}^{2} \prod_{j=1}^{r+1} \otimes \boldsymbol{I}_{n j} .
$$

By the orthogonal transformation defined in Section 2, we have

$$
\begin{aligned}
& \left(\boldsymbol{T}_{n_{1}} \otimes \cdots \otimes \boldsymbol{T}_{n_{r+1}}\right) \boldsymbol{V}\left(\boldsymbol{T}_{n_{1}} \otimes \cdots \otimes \boldsymbol{T}_{n r+1}\right)^{\prime} \\
= & \sum_{A=1}^{R} \sigma_{A}^{2} \prod_{j=1}^{r+1} n_{j}^{1-\delta s^{j}}\left[\prod_{t=1}^{r} \otimes\left(\boldsymbol{I}_{n_{t}}^{\boldsymbol{s}_{A}^{t}} \boldsymbol{H}_{n_{t}}^{1-\delta_{A}^{t}}\right)\right] \otimes \boldsymbol{H}_{n_{r+1}}+\sigma_{e}^{2} \prod_{j=1}^{r+1} \otimes \boldsymbol{I}_{n_{j}} \\
= & {\left[\sum_{P=1}^{R} \sigma_{P}^{2} \prod_{j=1}^{r+1} n_{j}^{1-\delta_{P}^{j}}+\sigma_{e}^{2}\right] \boldsymbol{I}_{n_{1}} \otimes\left(\prod_{t=2}^{r+1} \otimes \boldsymbol{H}_{n_{t}}\right) } \\
& +\sum_{A=1}^{R}\left[\sum_{P=A}^{R} \sum_{P \supset A} \sigma_{P}^{2} \prod_{j=1}^{r+1} n_{j}^{1-\delta_{P}^{j}}+\sigma_{e}^{2}\right] \boldsymbol{I}_{n_{1}} \otimes\left[\prod_{t=2}^{r} \otimes\left(\boldsymbol{I}_{n_{t}}^{\delta_{A-1}^{t}} \boldsymbol{K}_{n_{t}}^{\delta_{A-(A-1)}^{t}} \boldsymbol{H}_{n_{t}}^{1-\delta_{A}^{t}}\right)\right] \otimes \boldsymbol{H}_{n_{r+1}} \\
& +\sigma_{e}^{2} \boldsymbol{I}_{n_{1}} \otimes \cdots \otimes \boldsymbol{I}_{n_{r}} \otimes \boldsymbol{K}_{n_{r+1}} \\
= & B^{*} \boldsymbol{I}_{n_{1}} \otimes\left(\prod_{t=2}^{r+1} \otimes \boldsymbol{H}_{n_{t}}\right) \\
& +\sum_{A=1}^{R} B^{*}{ }_{(A)} \boldsymbol{I}_{n_{1}} \otimes\left[\prod_{t=2}^{r} \otimes\left(\boldsymbol{I}_{n_{t}}^{1-\delta_{A-1}^{t}} \boldsymbol{K}_{n_{t}}^{\delta_{A-(A-1)}^{t}} \boldsymbol{H}_{n_{t}}^{1-\delta_{A}^{t}}\right)\right] \otimes \boldsymbol{H}_{n_{r+1}} \\
& +\sigma_{e}^{2} \boldsymbol{I}_{n_{1}} \otimes \cdots \otimes \boldsymbol{I}_{n_{r}} \otimes \boldsymbol{K}_{n_{r+1}}
\end{aligned}
$$

where 


$$
\begin{aligned}
& B^{*}=\sum_{P=1}^{R} \sigma_{P} \prod_{j=1}^{r+1} n_{j}^{1-\delta_{P}^{j}}+\sigma_{e}^{2}, \\
& B^{*}{ }_{(A)}=\sum_{P=A}^{R} \sum_{\substack{P \supset A \\
F \subset R}} \sigma_{P}^{2} \prod_{j=1}^{r+1} n_{j}^{1-\delta_{P}^{j}}+\sigma_{e}^{2}, \quad A \subset R .
\end{aligned}
$$

From this it follows that

$$
\begin{aligned}
& A^{-1}=\prod_{j=2}^{r+1}\left(\frac{1}{n_{j}}\right) \frac{1}{B^{*}} \boldsymbol{I}_{n_{1}} \otimes \boldsymbol{E}_{n_{2}} \otimes \cdots \otimes \boldsymbol{E}_{n_{r}} \otimes \boldsymbol{E}_{n_{r+1}} \\
& +\sum_{A=1}^{R} \frac{1}{B^{*}{ }_{(A)}} \boldsymbol{I}_{n_{1}} \otimes\left[\prod_{t=2}^{r} \otimes\left\{\boldsymbol{I}_{n_{t}}^{\delta_{A-1}^{t}}\left(\boldsymbol{I}_{n_{t}}-\frac{1}{n_{t}} \boldsymbol{E}_{n_{t}}\right)^{\delta_{A-(A-1)}^{t}}\left(\frac{1}{\boldsymbol{n}_{t}} \boldsymbol{E}_{n_{t}}\right)^{1-\delta_{A}^{t}}\right\}\right] \otimes \frac{1}{n_{r+1}} \boldsymbol{E}_{n_{r+1}} \\
& \quad+\frac{1}{\sigma_{e}^{2}} \boldsymbol{I}_{n_{1}} \otimes \cdots \otimes \boldsymbol{I}_{n_{r}} \otimes\left(\boldsymbol{I}_{n_{r+1}}-\frac{1}{n_{r+1}} \boldsymbol{E}_{n_{r+1}}\right) \\
& =\prod_{j=2}^{r+1}\left(\frac{1}{n_{j}}\right) \frac{1}{B^{*}} \boldsymbol{I}_{n_{1}} \otimes \boldsymbol{E}_{n_{2}} \otimes \cdots \otimes \boldsymbol{E}_{n_{r}} \otimes \boldsymbol{E}_{n_{r+1}} \\
& \quad+\sum_{A=1}^{R} \frac{1}{B^{*}{ }_{(A)}} \boldsymbol{I}_{n_{1}} \otimes\left[\prod_{t=2}^{r} \otimes\left\{\boldsymbol{I}_{n_{t}}^{\delta_{A}^{t}} \boldsymbol{E}_{n_{t}}^{1-\delta_{A}^{t}}\right\}\right] \otimes \boldsymbol{E}_{n_{r+1}} \prod_{j=1}^{r+1}\left(\frac{1}{n_{j}}\right)^{1-\delta_{A}^{J}} \\
& \quad-\sum_{A=1}^{R} \frac{1}{B^{*}{ }_{(A)}} \boldsymbol{I}_{n_{1}} \otimes\left[\prod_{l=2}^{r} \otimes\left\{\boldsymbol{I}_{n_{t}}^{\delta_{A-1}^{t}} \boldsymbol{E}_{n_{t}}^{1-\delta_{A-1}^{t}}\right\}\right] \otimes \boldsymbol{E}_{n_{r+1}} \prod_{j=1}^{r+1}\left(\frac{1}{n_{j}}\right)^{1-\delta_{A-1}^{j}} \\
& \quad+\frac{1}{\sigma_{e}^{2}} \boldsymbol{I}_{n_{1}} \otimes \cdots \otimes \boldsymbol{I}_{n_{r}} \otimes\left(\boldsymbol{I}_{n_{r+1}}-\frac{1}{n_{r+1}} \boldsymbol{E}_{n_{r+1}}\right) .
\end{aligned}
$$

Then we have that the joint density function of all observations is given as follows :

$$
\begin{gathered}
f(\boldsymbol{x})=(2 \pi)^{-n_{1} \cdots n_{r+1} / 2} B^{*-1 / 2} \prod_{A \subset R}\left\{B^{*}{ }_{(A)}\right\}^{-\left(n_{1} \cdots n_{a-1}\right)\left(n_{a}-1\right) / 2}\left(\sigma_{e}^{2}\right)^{-n_{1} \cdots n_{r}\left(n_{r+1}-1\right) / 2} \\
\cdot \exp \left[-\frac{1}{2}\left\{\frac{\prod_{j=1}^{r+1} n_{j}}{B^{*}} \sum_{l_{1}}\left(\bar{x}_{l_{1}}-\mu\right)^{2}+\sum_{A=1}^{R} \frac{S_{(A)}^{*}}{B_{(A)}^{*}}+\frac{S_{e}}{\sigma_{e}^{2}}\right\}\right]
\end{gathered}
$$

where

$$
\begin{aligned}
& S_{(A)}^{*}=\prod_{j=a+1}^{r+1} n_{j} \sum_{t_{1}} \cdots \sum_{t_{a}}\left(\bar{x}_{t_{1} \cdots t_{a}}-\bar{x}_{t_{1} \cdots t_{\alpha-1}}\right)^{2}, \\
& S_{e}^{*}=\sum_{t_{1}} \cdots \sum_{t_{r+1}}\left(x_{t_{1} \cdots t_{r} t_{r+1}}-\bar{x}_{t_{1} \cdots t_{r}}\right)^{2} .
\end{aligned}
$$

Since it is seen that the family of the distributions of the sufficient statistics in our concern is complete, we have the same conclusion about the estimation problem for the nested design as we obtained in Theorem 4.3, 


\section{§. Acknowledgement.}

The author wishes his gratitude to Professor T. Kitagawa and Dr. A. Kudô of the Mathematical Institute, Kyushu University for helpful criticisms and encouragement throughout the course of writing this paper.

\section{KumAMoto UNIVERSITY and KYUSHU UNIVERSITY}

\section{References}

[1] N. FURUKAWA: The point estimation of the variance components in random effect model. Kumamoto Jour. of Science, 4 (1959), 111-133.

[2] N. FuкбкаWA: The point estimation of the parameters in the mixed model. Kumamoto Jour. of Science, 5 (1960), 1-43.

[3] F. A. Graybill and R. A. Huctquist: Theorems concerning Eisenhart's model II. Ann. Math. Stat., 32 (1961), 261-269.

[4] F. A. Graybill and A. W. Wortham: A note on uniformly best unbiased estimaters for variance components. Jour. Amer. Stat. Ass. 51 (1956), 266-268.

[5] W. Gadтschi: Some remarks on Herbach's paper, "Optimum nature of the F-test for Model II in the balanced case". Ann. Math. Stat., 30 (1959), 960-963.

[6] L. H. HERBACH: Properties of Model II-type analysis of variance test, A; Optimum nature of the F-test for Model II in the balanced case. Ann. Math. Stat., 30 (1959), 939-959.

[7] E. L. Lermann: Notes on the theory of estimation. Associated Student's Store, Univ. of California (1950).

[8] E. L. Lehmann and H. Scheffe: Completeness, similar regions and unbiased estimation, Part I. Sankhya, 10 (1950), 305-340.

[9] E. L. Lehmann and H. Scheffe: Completeness, similar regions and unbiased estimation, Part II. Sankhya, 15 (1956), 219-236.

[10] S. N. Roy and R. Gnanadesikan: Some contributions to ANOVA in one or more dimensions; I. Ann. Math. Stat. 30 (1959), 304-317.

[11] D. L. WeEks and F. A. Graybill: A minimal sufficient statistics for general class of designs. Sankhya, Ser. A, 24 (1962), 339-354.

This paper is in part supported by a grant given to the Kyushu University from U. S. Public Health Service (Gm 09470-02) (Chief investigator Prof. Dr. K. Yamaoko). 